\title{
Nanoscale Morphology of Apatite Precipitated onto Synthetic Hydroxyapatite from Simulated Body Fluid
}

\author{
Jennifer Vandiver ${ }^{1, a}$, Nelesh Patel
Ortiz $^{2, b}, d^{d^{*}}$ William Bonfield
}

${ }^{1}$ Department of Materials Science and Engineering, RM 13-4022, 77 Massachusetts Avenue, Cambridge, Massachusetts 02139 USA

${ }^{2}$ Department of Materials Science and Metallurgy, University of Cambridge, Pembroke Street, Cambridge CB2 3QZ U.K.

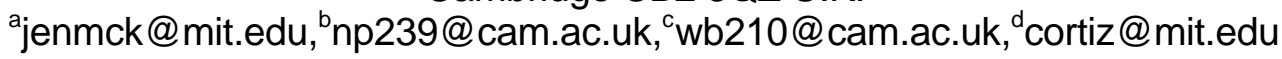

Keywords: Hydroxyapatite, Atomic Force Microscopy (AFM), Simulated Body Fluid (SBF), Apatite

Abstract. Dense, polycrystalline, synthetic hydroxyapatite (HA) was incubated for 36 days in modified simulated body fluid (SBF) with increased $\mathrm{HCO}_{3}{ }^{-}$and reduced $\mathrm{Cl}$ ion concentrations (27 and $120 \mathrm{mM}$, respectively) closer to actual blood plasma than typical SBF. The resulting precipitated apatite layer was characterized by X-ray photoelectron spectroscopy (XPS) and contact angle measurements and found to be nonstoichiometric, calcium deficient $(\mathrm{Ca} / \mathrm{P} \sim 1.06)$, noncarbonate containing, and of intermediate hydrophilicity (advancing contact angle, $\theta_{\mathrm{a}}=76.5 \pm 1.3^{\circ}$ ). The nanoscale surface topography of the SBF-incubated HA sample was imaged by tapping mode atomic force microscopy (TMAFM), observed to be $=100 \mathrm{~nm}$ in thickness, and composed of three distinct morphologies. These topographically distinct regions were localized within individual grains and facets of the initial HA surface and included: hemispherical, globular structures (maximum lateral dimension, $d=44.7 \pm 12.7 \mathrm{~nm}$, peak-to-valley height, $h=3.6 \pm 2.7 \mathrm{~nm}$ ); elongated, needle- like structures (minimum lateral dimension, $w=31.0 \pm 8.5 \mathrm{~nm}, d=104.4 \pm 31.1 \mathrm{~nm}, h=5.0 \pm 3.2$ $\mathrm{nm}$ ), and regions of larger, irregularly shaped structures that were relatively smooth $(d=504.9 \pm 219.1 \mathrm{~nm}, h=104.0 \pm 51.7 \mathrm{~nm})$.

\section{Introduction}

Synthetic hydroxyapatite (HA, $\left.\mathrm{Ca}_{5}\left(\mathrm{PO}_{4}\right)_{3} \mathrm{OH}\right)$, HA-based biomaterials, and HA coatings are used extensively for hard tissue applications due to their bioactivity [1]. Upon implantation in vivo [2] or incubation in vitro in simulated body fluid (SBF) [3], an apatite layer forms on the surface which is considered essential for the nucleation of biological apatite, the promotion of protein adsorption and cell adhesion, and ultimately, the creation of a strong bond with the surrounding tissue [4]. The objective of this study was to directly visualize and quantify the nanoscale topography of apatite precipitated in vitro from SBF onto dense, polycrystalline, phase pure HA using tapping mode atomic force microscopy (TMAFM) imaging, which enables spatial resolutions of $<1 \mathrm{~nm}$. New information is presented on the morphological heterogeneity of the apatite layer, as well as the nature of the transition boundaries between topographically different regions. Such a methodology has great potential to contribute insights into the physiochemical mechanisms and temporal evolution of HA interfacial apatite layers and molecular origins of their bone bonding capability.

\section{Materials and Methods}

HA Sample Preparation, Characterization, and Incubation in SBF. Synthetic, phase pure, dense, polycrystalline HA pellets $(\sim 1 \mathrm{~cm}$ in diameter) were prepared by an aqueous precipitation reaction between calcium hydroxide and phosphoric acid as described previously [5]. The pellets were found to be $>98 \%$ of the theoretical density $\left(3.13 \pm 0.015 \mathrm{~g} / \mathrm{cm}^{3}\right)$, highly crystalline, phase pure, and approximately stoichiometric $(\mathrm{Ca} / \mathrm{P}$ ratio $=1.67)$ as measured by water displacement, wide 
angle X-ray diffraction, and X-ray fluorescence [6]. SBF (total ionic strength $=0.155 \mathrm{mM}, \mathrm{pH} 7.4$ ) was prepared with the following ion concentrations $(\mathrm{mM}) ; \mathrm{Na}^{+}(142), \mathrm{K}^{+}(5), \mathrm{Mg}^{2+}(1.5), \mathrm{Ca}^{2+}(2.5)$, $\mathrm{Cl}^{-}(120), \mathrm{HCO}_{3}{ }^{-}(27), \mathrm{HPO}_{4}{ }^{2-}(2.27), \mathrm{SO}_{4}{ }^{2-}(0.5)[7] . \mathrm{NaCl}(6.55 \mathrm{~g}), \mathrm{NaHCO}_{3}(2.27 \mathrm{~g}), \mathrm{KCl}(0.373 \mathrm{~g})$, $\mathrm{Na}_{2} \mathrm{HPO}_{4} \cdot 2 \mathrm{H}_{2} \mathrm{O}(0.178 \mathrm{~g}), \quad \mathrm{MgCh} \cdot 6 \mathrm{H}_{2} \mathrm{O}(0.305 \mathrm{~g}), 37 \quad \mathrm{wt} \% \quad \mathrm{HCL} \quad(5 \mathrm{ml}), \quad \mathrm{CaCb}(0.278 \mathrm{~g})$, $\mathrm{NaSO}_{4}(0.071 \mathrm{~g})$, and $\mathrm{NH}_{2} \mathrm{C}\left(\mathrm{CH}_{2} \mathrm{OH}\right)_{3}$ (Tris buffer,6.055g) were dissolved in that order into $500 \mathrm{~mL}$ Millipore water at $37^{\circ} \mathrm{C}$ under continuous magnetic stirring. Millpore water was added to increase the total volume to $1 \mathrm{~L}$, the temperature was returned to $37^{\circ} \mathrm{C}$, and the $\mathrm{pH}$ was balanced to 7.4 using $1 \mathrm{M}$ HCL. The solution was filtered with a $0.22 \mu \mathrm{m}$ vacuum filter and stored in the refrigerator at $4^{\circ} \mathrm{C}$ until use. The HA pellets were incubated in $100 \mathrm{ml}$ of $\mathrm{SBF}$ at $37^{\circ} \mathrm{C}$ for 36 days with no refreshing of the solution.

XPS and Contact Angle Measurements. A Kratos AXIS Ultra Imaging X-ray Photoelectron Spectrometer with AlK $\alpha$ X-ray source was used to analyze the initial HA and the precipitated apatite surface compositions at a take-off angle of $0^{\circ}$ (penetration depth $<10 \mathrm{~nm}$ ). Advancing contact angles $\left(\theta_{\mathrm{a}}\right)$ were measured using deionized water to assess the wettability of the initial and SBF-incubated HA surfaces (Video Contact Angle System 2000, AST Inc.).

Atomic Force Microscopy (AFM). Contact mode AFM (CMAFM) was used to measure the initial HA grain size and TMAFM was employed to image surface topography at higher resolutions, both in ambient conditions with a Digital Instruments Nanoscope IIIA System Controller and Multimode AFM. Thermomicroscopes $\mathrm{Si}_{3} \mathrm{~N}_{4} \mathrm{~V}$-shaped cantilever (end radius, $\mathrm{R}_{\mathrm{TIP}}<50 \mathrm{~nm}$, spring constant, $\mathrm{k}_{\mathrm{c}} \sim 0.01 \mathrm{~N} / \mathrm{m}$ ) and Olympus AC240TS-2 rectangular Si cantilevers $\left(\mathrm{R}_{\mathrm{TIP}}<10 \mathrm{~nm}, \mathrm{k}_{\mathrm{c}}=2 \mathrm{~N} / \mathrm{m}\right)$ were used for CMAFM and TMAFM, respectively. Height images were employed to quantify the dimensions of topographical features.

\section{Results}

XPS and Contact Angle Measurements.

An XPS survey spectrum on the SBF- Fig. 1. XPS spectrum of HA with precipitated apatite layer incubated sample showed the expected $\mathrm{Ca}(2 \mathrm{~s}, 3 \mathrm{~s}, 2 \mathrm{p}), \mathrm{P}(2 \mathrm{~s}, 2 \mathrm{p})$, and $\mathrm{O}($ Auger, $1 \mathrm{~s}, 2 \mathrm{~s})$ peaks, confirming that the precipitated surface layer was indeed apatite (Fig. 1). Small peaks of Fe, a contaminant from SBF reagents, as well as $\mathrm{N}$ and $\mathrm{Na}$ directly from SBF reagents, were noted. $\mathrm{Na}^{+}$substitution is a possibility. A high resolution scan of the $\mathrm{C} 1 \mathrm{~s}$ peak revealed typical hydrocarbon contamination and the absence of a carbonate peak at $289.3 \mathrm{eV}$. The $\mathrm{Ca} / \mathrm{P}$ ratio was calculated to be 1.06 , i.e. Ca-deficient. $\theta_{\mathrm{a}}$ was found to be $76.5 \pm 1.3^{\circ}$ for the SBF-

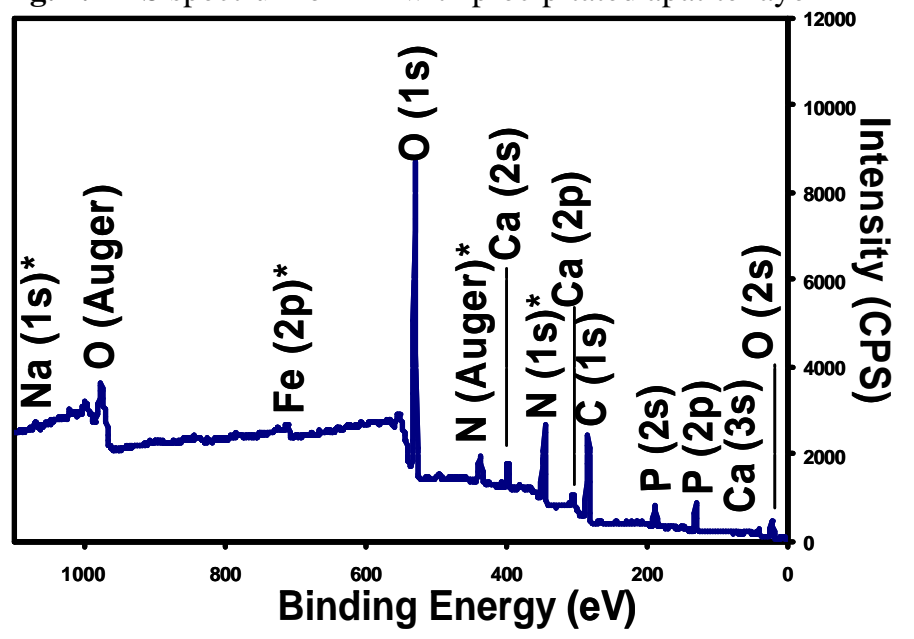
incubated sample, as compared to $65.2 \pm 0.85^{\circ}$ for the initial HA surface $(\mathrm{p}<0.005)$.

Surface Morphology of Precipitated Apatite Layer. The original HA surface was composed of smooth faceted grains with a maximum dimension, $d$, of $1.17 \pm 0.76 \mu \mathrm{m}$, as determined via CMAFM height images (data not shown). Fig. 2a and b compare TMAFM deflection images of the initial HA and SBF-incubated HA surfaces, respectively. The initial HA surface was found to have an intergrain (across grain boundary) peak-to-valley height, $h$, of $119.84 \pm 74.81 \mathrm{~nm}$ and an intragrain peak-to-valley feature height, $h$, of $<1 \mathrm{~nm}$. The underlying HA grain boundaries were still visible after SBF incubation and the SBF-incubated HA surface showed grains with $d=1.05 \pm$ $0.47 \mu \mathrm{m}$, statistically similar to the initial HA grain size $(\mathrm{p}<0.05)$. Linear height profiles of "bare" HA regions adjacent to apatite regions, which were rarely found, showed the apatite layer thickness 
in these areas to be $70.6 \pm 49.9 \mathrm{~nm}$. A distinct change in intragrain topography was observed due to precipitation of an apatite layer compared to the original HA surface (Fig. 2b). In particular, three

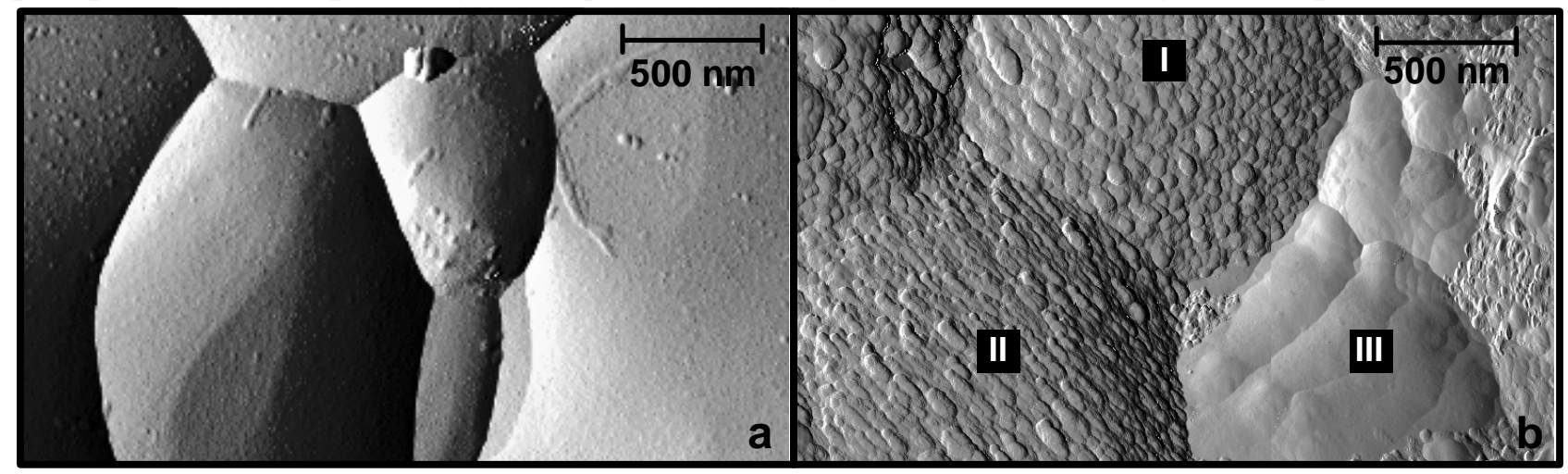

Fig. 2. TMAFM deflection images of (a) initial HA surface and (b) HA surface after 36 days incubation in SBF showing three distinct morphologies labeled I (hemispherical, globular), II (elongated, needle-like), and III (large, irregular, smooth).

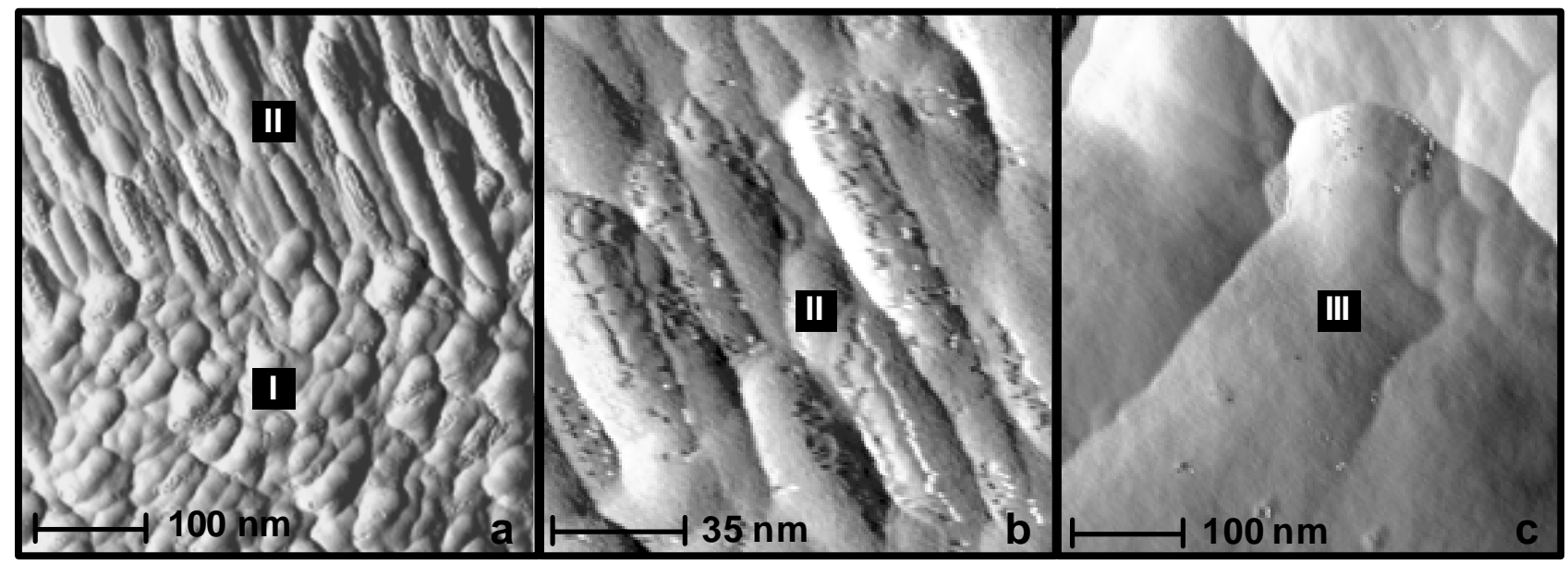

Fig. 3. TMAFM deflection images of precipitated apatite layer; (a) the interface between regions I (hemispherical, globular) and II (elongated, needle-like), (b) region II at higher resolutions, and (c) region III (large, irregular, smooth).
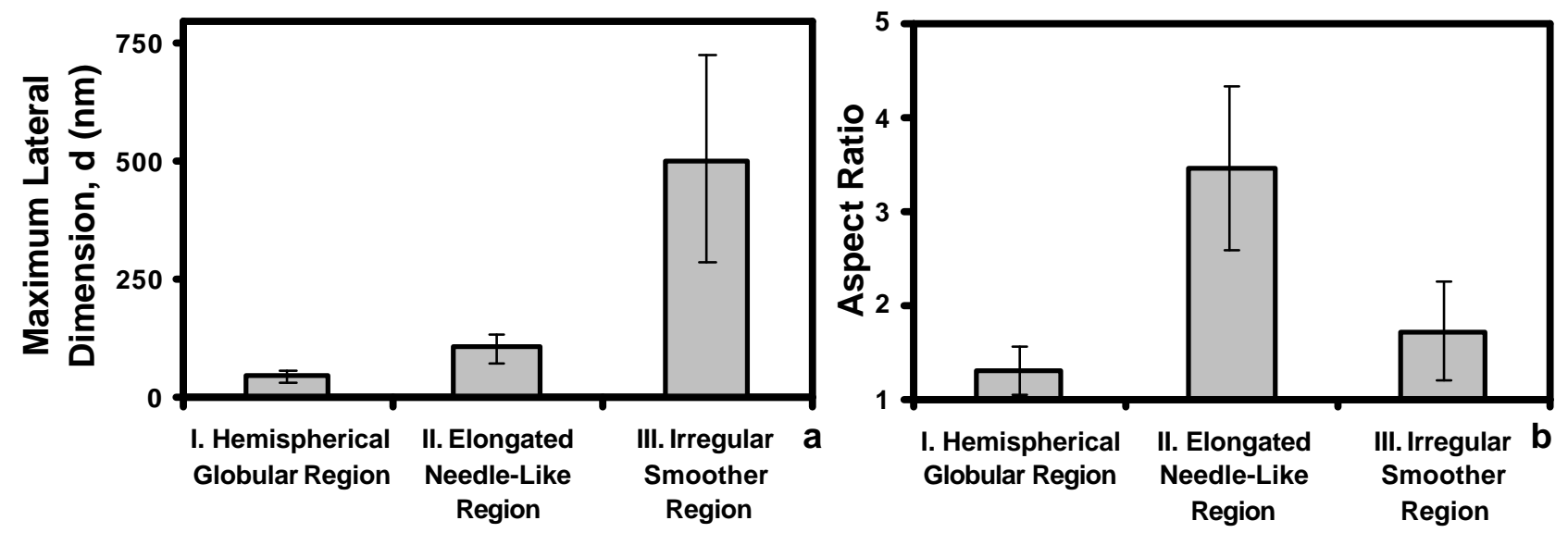

Fig. 4. Average maximum lateral dimensions and aspect ratios of topographical features in three morphological regions taken from TMAFM height images; hi-lo bars are standard deviations for a minimum of 9 measurements per dataset.

distinct morphologies were visualized; I) hemispherical, globular structures $(d=44.7 \pm 12.7 \mathrm{~nm}$, $h=3.6 \pm 2.7 \mathrm{~nm}$ ), II) elongated, needle-like structures (width, $w=31.0 \pm 8.5 \mathrm{~nm}, d=104.4 \pm 31.1 \mathrm{~nm}$, $h=5.0 \pm 3.2 \mathrm{~nm}$ ), and III) larger, irregularly shaped structures with relatively smooth surfaces $(d=504.9 \pm 219.1 \mathrm{~nm}, h=104.0 \pm 51.7 \mathrm{~nm})$. As shown in Fig. 2b, each of these morphologies was localized within individual grains or facets. Fig. 3 shows higher resolution images of these three 
morphological regions. Finer nanoscale topographical features, exposing a thin surface layer, were observed within individual hemispherical globules (I, Fig. 3a) and needle-like precipitates (II, Fig. 3b). Fig. 4 is a statistical analysis of the lateral topographical dimensions of each of the three morphologies and showed statistically significant differences between all datasets $(\mathrm{p}<0.01)$.

\section{Discussion}

The thinness of the apatite layer observed in this study compared to other reports on HA granules, pellets, and coatings [3,8] may be accounted for by a number of factors, including the lower solubility of fully dense, stoichiometric, phase pure HA (which is known to reduce the rate of apatite precipitation [2], the decrease in $\mathrm{Ca}^{2+}$ ions in solution with immersion time, and the unique $\mathrm{SBF}$ formulation used here which has increased $\mathrm{HCO}_{3}{ }^{-}$and reduced $\mathrm{Cl}$ ion concentrations which are closer to those found in blood plasma. Previous XPS studies on apatite layers precipitated on $\mathrm{HA}$ in SBF have found them generally to be $\mathrm{Ca}$ deficient $(\mathrm{Ca} / \mathrm{P}=1.25-1.66)[9,10]$. The absence of carbonate was consistent with previous reports on apatite precipitated onto well-crystallized HA in SBF [9]. Other in vitro studies of apatite precipitation in $\mathrm{SBF}$ with increased $\mathrm{HCO}_{3}{ }^{-}$ions show an apatite layer of elongated nodules, which is less porous than an analogous apatite layer precipitated from SBF with lower concentrations of $\mathrm{HCO}_{3}{ }^{-}$ions [11]. In vivo experiments on plasma-sprayed HA coatings show apatite crystallites at the HA-bone interface 3-7 X 30-40 X 60-100 nm in size [12], consistent with the dimensions of the needle-like elongated structures observed in this study. Transitions between the different surface morphologies appear to be located at the underlying grain boundaries or possibly underlying facets. Different facets or grains on the initial HA surface would have different exposed crystal planes and therefore differing solubility, which has been shown to have a large influence on the structure, morphology, and composition of the apatite layer [13]. Additionally, previous work has shown surface charge of the initial HA surface to vary with nanoscale position on the surface and across grain boundaries, likely associated with exposed crystal plane [6], which could also affect apatite layer precipitation. Future experiments include spatially and chemically specific force spectroscopy on the three distinct morphological regions.

\section{Acknowledgements}

The authors would like to thank the MIT Center for Materials Science and Engineering, the NanoMechanical Technology Laboratory in the Department of Materials Science and Engineering, James Holder (University of Cambridge, UK), and Laurel Ng (MIT) for training, assistance and facilities. Funding was provided by the Cambridge - MIT Institute (CMI).

\section{References}

[1] L.L. Hench: J Am Ceram Soc; 81(7) (1998), p. 1705.

[2] M. Neo, T. Nakamura, C. Ohtsuki, T. Kokubo, T. Yamamuro: J Biomed Mater Res; 27 (1993), p. 999.

[3] J.H.C. Lin, K.H. Kuo, S.J. Ding, C.P. Ju: J Mater Sci: Mater Med; 12 (2001), p. 731.

[4] T. Kokubo, H. Kushitani, S. Sakka: J Biomed Mater Res; 24 (1990), p. 721.

[5] M. Akao, H. Aoki, K. Kato: J Mater Sci; 16 (1981), p. 809.

[6] J. Vandiver, D. Dean, N. Patel, B. W., C. Ortiz: Accepted to Biomaterials; (2004), p.

[7] A.C. Tas: Biomaterials; 21 (2000), p. 1429.

[8] K.A. Khor, H. Li, P. Cheang: Biomaterials; 24 (2003), p. 769.

[9] S.R. Radin, P. Ducheyne: J Biomed Mater Res; 27 (1993), p. 35.

[10] N. Kanzaki, K. Onuma, A. Ito, K. Teraoka, T. Tateishi, S. Tsutsumi: J. Phys. Chem. B; 102 (1998), p. 6471.

[11] E.I. Dorozhkina, S.V. Doroshkin: Colloids and Surf A; 210 (2002), p. 41.

[12] A.E. Porter, L.W. Hobbs, V.B. Rosen, M. Spector: Biomaterials; 23 (2002), p. 725.

[13] A.E. Porter, N. Patel, J.N. Skepper, S.M. Best, W. Bonfield: Biomaterials; 24 (2003), p. 4609. 